\title{
Multiscale Snapshots: Visual Analysis of Temporal Summaries in Dynamic Graphs
}

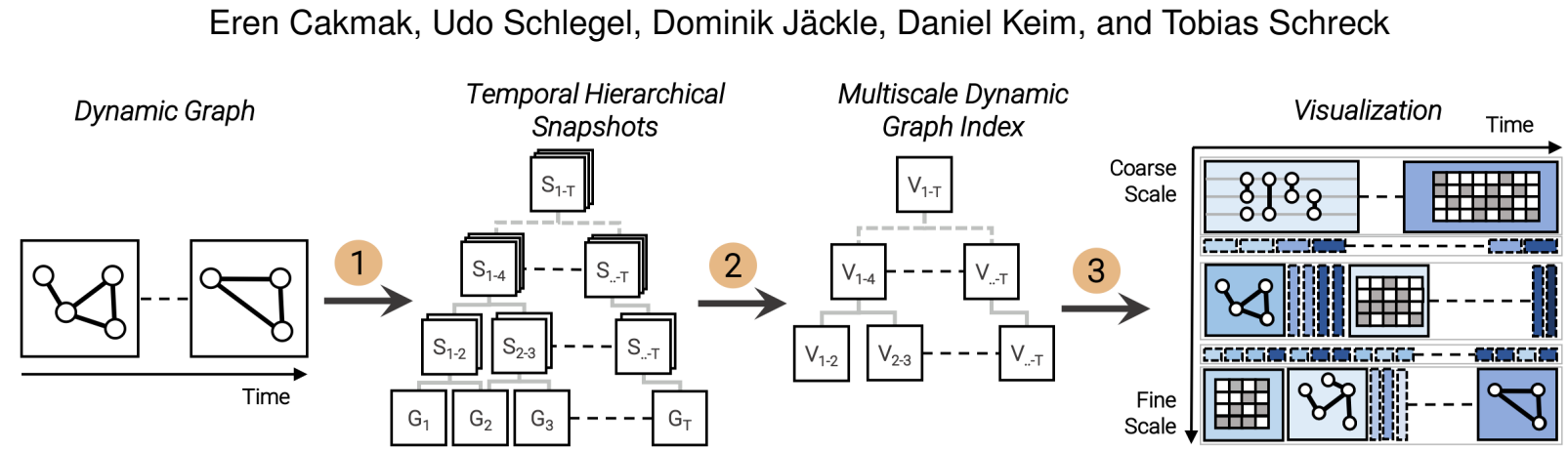

1) Temporal Summaries

2) Graph Embedding

3) Visual Mapping

Fig. 1. Overview of our Multiscale Snapshots approach: We (1) recursively create temporal summaries (snapshots) of graphs at different temporal scales (time granularities); (2) We then apply an unsupervised graph learning method (graph embedding) to learn low-dimensional representations of snapshots; Then, we (3) then give an exploratory visualization that organizes the snapshots of different temporal granularities in a hierarchy, to provide an overview of the evolving structural properties, which utilizes the graph embeddings for analytical tasks (e.g., similarity search). The approach allows rich interaction with the temporal and structural aggregates to understand large time-dependent graphs, for example, to visually analyze reoccurring temporal states.

\begin{abstract}
The overview-driven visual analysis of large-scale dynamic graphs poses a major challenge. We propose Multiscale Snapshots, a visual analytics approach to analyze temporal summaries of dynamic graphs at multiple temporal scales. First, we recursively generate temporal summaries to abstract overlapping sequences of graphs into compact snapshots. Second, we apply graph embeddings to the snapshots to learn low-dimensional representations of each sequence of graphs to speed up specific analytical tasks (e.g., similarity search). Third, we visualize the evolving data from a coarse to fine-granular snapshots to semi-automatically analyze temporal states, trends, and outliers. The approach enables us to discover similar temporal summaries (e.g., reoccurring states), reduces the temporal data to speed up automatic analysis, and to explore both structural and temporal properties of a dynamic graph. We demonstrate the usefulness of our approach by a quantitative evaluation and the application to a real-world dataset.
\end{abstract}

Index Terms-Dynamic Graph, Dynamic Network, Unsupervised Graph Learning, Graph Embedding, Multiscale Visualization.

\section{INTRODUCTION}

A dynamic graph models changing relationships between entities over time. Many real-world data analysis problems rely on dynamic graphs, including, among others, social, computer, and communication networks, and in practice, contain large amounts of dynamic data, hence presenting challenges for effective exploration. An important task in such dynamic graphs is to obtain an overview of the evolving topology by identifying meaningful temporal intervals and their underlying changing structural properties [22]. For instance, analysts are often interested in the identification of stable, reoccurring, transition, and outlier states [61]. However, as dynamic graphs are often large-scale and evolve over long periods, it is a major challenge to identify suitable analysis methods and present the data in a readable, scalable, and expressive manner [13]. Previous approaches for visual analysis of dynamic graph data, therefore, often incorporate temporal abstraction

- Eren Cakmak, Udo Schlegel, and Daniel Keim are with the University of Konstanz, Germany. E-mail: firstname.lastname@uni-konstanz.de

- Dominik Jäckle is an Independent Researcher, Munich, Germany. E-Mail:dominikjaeckle@gmail.com

- Tobias Schreck is with the TU Graz, Austria.

E-Mail: tobias.schreck@cgv.tugraz.at

Manuscript received xx xxx. 201x; accepted xx xxx. 201x. Date of Publication xx xxx. 201x; date of current version xx xxx. 201x. For information on obtaining reprints of this article, please send e-mail to: reprints@ieee.org. Digital Object Identifier: $x x . x x x x / T V C G .201 x . x x x x x x x$ methods (e.g., temporal aggregation and dimensionality reduction) to provide an overview of higher-level structures over time [61]. In real-world applications, the usefulness of such temporal abstraction methods depends on many factors, including the selection of an appropriate temporal scale, the user task at hand, graph size, and frequency of topological changes. Currently, visual analytics systems for dynamic graphs lack methods for the visual analysis of dynamic processes at different temporal abstraction scales (multiscale analysis), often leaving the analyst with the challenging task of distinguishing overlapping temporal changes manually.

We propose Multiscale Snapshots, a novel visual analytics approach to semi-automatically provide a multiscale overview of structural and temporal changes in dynamic graphs. We combine temporal hierarchical abstractions with unsupervised graph learning methods to enable the identification of similar evolving graphs. First, the temporal hierarchical snapshots summarize the dynamic graph recursively at multiple temporal scales to reduce the size of the large-scale data. Second, we apply unsupervised graph learning (e.g., graph2vec [50]) to the snapshots of the hierarchy to learn low-dimensional representations of graph sequences, which enables users to use the embeddings for analytical tasks (e.g., similarity search) and later on to adapt the temporal scale semi-automatically. Third, the visualization of the hierarchy of snapshots provides an overview of trends, allows users to compare periods, and to explore structural as well as temporal properties of dynamic graphs. The approach enables exploring various abstraction methods at multiple temporal scales to provide an overview of large dynamic graphs temporal and structural properties. 
With Multiscale Snapshots, we can retrace how dynamic patterns and changing graph properties affect the overall evolving data, and compare temporal structures at different levels of temporal resolution. The contributions of this work are the following: (1) The Multiscale Snapshots approach to visually analyze temporal and structural similarities at multiple temporal scales; (2) A temporal hierarchical abstraction using unsupervised graph learning methods to reduce the size of dynamic graphs and speed up analytical tasks (e.g., similarity search).

The remainder of this paper is structured as follows. Section 2 discusses the related work. Section 3 describes the addressed problems, the research gap, and the design goals. In Section 4, we describe the Multiscale Snapshots approach and our implementation. In Section 5 , we quantitatively evaluate and apply the approach to a real-world, large-scale dynamic graph. A discussion is given in Section 6, before Section 7 concludes.

\section{Related Work}

Multiscale Snapshots combines temporal summaries with graph embeddings to present an overview of the underlying dynamic phenomena. In the following, we discuss related work from automated analysis, visualization, visual analytics, and multiscale visualization approaches for dynamic graphs.

\subsection{Dynamic Graph Analysis and Visualization}

The visual analysis of long graph sequences has lately gained research attention [13]. The automatic analysis, such as the temporal analysis of static as well as dynamic graph metrics (e.g., centrality, diameter [19], or change centrality |33|), enables us to examine structural properties of the data. Furthermore, recent approaches in unsupervised learning focus on embedding graph structures into low-dimensional space [66] However, only analyzing such automatically extracted structural properties might hide specific local dynamic changes (e.g., changes of density) and may fail to capture the overall dynamic phenomena [66] Interactive visualizations can overcome these challenges by allowing analysts to explore the dynamic relationships in their evolving structural context, and several visualization techniques for dynamic graphs have been proposed. Popular approaches display dynamic graphs as animations [8 30, 54], timeline [11, 28, 29, 38, 65] and hybrid visualizations [9 23.56]. For further reading, we refer to the surveys of Kerracher et al. [44], Beck et al. [13], and Nobre et al. [52].

Many of the proposed visualization techniques, however, do not scale to a large number of nodes, edges, and time steps at the same time [37]. Consequently, to adapt existing techniques to large-scale dynamic graphs, visual analytics approaches were proposed that combine automatic analysis methods with interactive visualizations to reduce the presented data and highlight structural changes.

\subsection{Visual Analytics of Dynamic Graphs}

The visual analytics of dynamic graphs aims to seamlessly integrate graph analysis methods [51] with visualization techniques [13] to interactively analyze the evolving structural properties. Such visual analytics approaches facilitate abstraction methods for large-scale dynamic graphs to reduce the amount of data and provide an overview of high-level changes. The usefulness of such abstraction methods, however, depends on the applied method (e.g., temporal clustering) and input parameters (e.g., number of clusters) [38]. Therefore, according to Aigner et al. [4], it is essential to interactively adapt abstraction methods and tune their underlying parameters to identify changes that otherwise would remain hidden.

In general, there are two categories of abstraction methods; data space abstraction (e.g., sampling, clustering) and visual space abstraction (e.g., zooming, focus-and-context) [27]. The data space abstraction in dynamic graphs reduces the number of graph elements or time steps [57]. Often, data space abstraction methods lower the resolution of the data (e.g., temporal aggregation [49]). For instance, Van den Elzen et al. [61] proposed a visual analytics approach that segments and aggregates sequences of graphs to a vector and applies dimensionality reduction to obtain an overview of the states in dynamic graphs. However, the resulting overview depends strongly on the selected segmentation scale and the abstraction method (extracted features) into vectors. Further, the dimensionality reduction technique is hard to interpret as the projection does not visualize the evolving graph structure. For an overview of data space abstraction methods, we recommend the recent survey of Liu et al. [47]. The visual space abstraction methods in dynamic graphs reduce the amount of presented data (e.g., by applying zooming [14]). Many of the visual space abstraction methods allow the user to interactively change the depicted

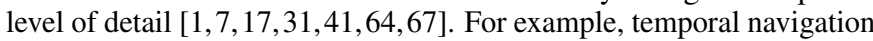
methods help to interactively adapt the horizontal (e.g., TempoVis [3]) and vertical (e.g., Bender-deMoll and McFarland [16]) time dimension. Multiple visual analytics approaches, including visual abstraction methods, were recently proposed. For instance, Small MultiPiles [9] enables users to interactively stack and present a sequence of graphs as piles of adjacency matrices to reduces the number of displayed views. Furthermore, Cubix [10] allows users to visually explore adjacency matrices of dynamic graphs in a cube metaphor. However, in both approaches, the identification of temporal patterns in long sequences of adjacency matrix visualizations remains challenging due to limited display space and overlapping issues in $3 \mathrm{D}$ visualizations.

Many of the previously proposed approaches focus mainly on aggregation and display the abstracted temporal or structural dimension at one scale, which makes it challenging to investigate the influences of abstraction methods on the resulting visualization, as patterns may be found at different scales and intervals. Multiscale visualizations aim to overcome these challenges by simultaneously displaying different levels of abstraction, hence providing an encompassing overview of possible structural and temporal aggregation levels.

\subsection{Multiscale Dynamic Graph Visualizations}

Multiscale (multiresolution) visualizations present the data at multiple user-defined levels of abstraction and are useful to set detailed abstraction levels into the overall temporal context [32]. For example, Javed and Elmqvist [42] stack different levels of zoomed time-series data in a tree structure to serve as a graphical history and preserve the context during zooming. Nearly all of the previously mentioned approaches visualize the dynamic graphs on a single time granularity (scale) using mostly one adjustable abstraction method. One notable exception is the recent work of Burch and Reinhardt [22] that proposed a timeline visualization technique that allows exploring dynamic graphs at different temporal granularities. However, the approach focuses on bipartite graphs, and due to the overplotting produced by the interleaving method, the identification of temporal patterns remains challenging.

Most of the listed approaches for dynamic graphs focus on the analysis of dynamic graphs at a particular temporal scale and require the manual definition of parameters. For instance, the work of Van den Elzen et al. [61] requires users to define a discretization scale, feature selection, and the choice of a suitable dimensionality reduction technique. In contrast to these approaches, we propose using temporal hierarchical abstractions with unsupervised learning methods to explore input parameters (e.g., discretization scale) and simultaneously visualize graph sequences at different levels of temporal abstraction.

\section{Application Background}

The analytical goal of our approach is to provide an overview of evolving graph properties at multiple abstraction scales. In the following, we describe the addressed problems, the research gap we close, and our derived design goals.

\subsection{Problem Description}

The starting point of data analysis is often an overview visualization to examine the overall data structure, and to identify useful analytical and visualization techniques [58]. However, providing an overview of largescale dynamic graphs can be challenging for multiple reasons [13]. First, the complexity and size of data pose various challenges as many dynamic graph visualization techniques do not scale [12]. Second, it is challenging to visualize dynamic graphs as there is a trade-off between displaying the detailed graph structure for each time step, and also presenting the evolving properties over time. For instance, animations 


\begin{tabular}{|c|c|c|c|c|c|c|c|c|c|c|c|c|}
\hline \multirow[t]{2}{*}{ Publication, Year } & \multicolumn{3}{|c|}{ Visualization } & \multicolumn{3}{|c|}{ Scalability } & \multicolumn{6}{|c|}{ Temporal Explorability } \\
\hline & 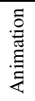 & 竭 & $\begin{array}{l}\text { 를 } \\
\text { 齐 }\end{array}$ & $\begin{array}{l}\overline{\text { ज्ञg }} \\
\text { क }\end{array}$ & 嘿 & 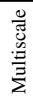 & $\begin{array}{l}\frac{\tilde{q}}{0} \\
z\end{array}$ & $\begin{array}{l}\mathscr{8} \\
\vec{\Phi} \\
\vec{\Phi}\end{array}$ & 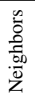 & $\frac{n}{\tilde{E}}$ & 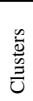 & 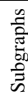 \\
\hline Multiscale Snapshots & & & $\bullet$ & $\bullet$ & $\bullet$ & $\bullet$ & $\bullet$ & $\bullet$ & $\bullet$ & & $\bullet$ & $\bullet$ \\
\hline Rufiange \& McGuffin 56, 2013 & & & $\bullet$ & - & & & $\bullet$ & $\bullet$ & $\bullet$ & $\bullet$ & & \\
\hline Hadlak et al. 38,2013 & & $\cdot$ & & - & & - & & & & & • & $\cdot$ \\
\hline Cui et al. 2014 & & • & & - & • & & & & • & & • & \\
\hline Bach et al. 9,2015 & & & $\bullet$ & • & & & & & & & • & • \\
\hline Burch \& Weiskopf 233, 2015 & & & $\bullet$ & • & & & $\bullet$ & • & • & & • & \\
\hline Bach et al. 11,2015 & & • & & • & • & - & & & & & & \\
\hline Van den Elzen et al. 61,2016 & & • & & $\bullet$ & $\bullet$ & & $\bullet$ & $\bullet$ & $\bullet$ & & & \\
\hline Burch et al. 22, 2017 & & - & & • & & - & & • & & & • & \\
\hline Dal et al. 29,2017 & & • & & - & & & $\bullet$ & & • & & & \\
\hline Xu et al. 65,2018 & & - & & • & & & $\bullet$ & • & & & - & $\cdot$ \\
\hline Wang et al. 63,2019 & & - & & - & & & $\bullet$ & • & $\bullet$ & - & & \\
\hline
\end{tabular}

Table 1. The comparison highlights the essential temporal properties of related visualization techniques, ordered by publication date, and assessed by us to the best of our knowledge from analysis of the works. The visualization category classifies the techniques using the taxonomy of Beck et al. [13]. The scalability category elaborates on multiscale temporal approaches and the temporal scalability with large scalability meaning a dynamic graph with more than 1000 graphs. The temporal explorability is adapted from the work of Nobre et al. [52] and illustrates whether the graph structures (e.g., neighbors, clusters) are explorable and comparable within the temporal dimension.

support the exploration of each static graph over time. However, animations are considered unsuited to provide an overview of long periods due to the problems caused by cognitive effort [8, 40 , 60] and difficulties maintaining a mental map in dynamic graphs [54]. Third, creating compact temporal abstractions (summaries) of dynamic graphs is user task-dependent and relies upon the application domain as well as data properties. For example, a fine-grained temporal aggregation in large-scale dynamic graphs results in various intervals with little information unable to provide an overview [16]. In contrast, coarse-scale aggregation produces only a few intervals, which may contain a high variance, where meaningful intervals could go unnoticed. Finding appropriate levels of abstraction is a non-trivial task [45].

\subsection{Gaps in Related Approaches}

We compare a selection (see Table 1) of recent work on dynamic graph visualization to point out the gap we intend to close. The selected publications are based on the recursive search of references from the recent surveys of Beck et al. [13] and Nobre et al. [52]. The categories of our comparison comprise visualization techniques from the dynamic graph taxonomy [13], the temporal scalability, including multiscale approaches, and the temporal explorability of different graph structures [52].

The comparison reveals several insights. First, the number of temporal multiscale approaches for dynamic graphs is limited. Multiscale approaches present either time series in a multiresolution design (e.g., graph metrics [38]) or include visualizations having multiscale characteristics (e.g., time curves [11]). Second, timeline visualizations (time-to-space mappings) reduce the size of the data and are suitable to provide an overview of a sequence of graphs. However, such approaches abstract and discretize structural information at one temporal scale (uniform time slicing), often requiring users to manually identify overlapping temporal patterns [63]. For example, timeline-based visualizations often require to define input parameters, e.g., discretization parameters and derived features [61]. Third, current work either introduce a single complex visualization, or the combination of various simple views, which poses the question of how to arrange the different visualizations in the limited screen space and link them effectively.

In summary, a significant number of visualization techniques view a dynamic graph as a series of static graphs which neglects to simultaneously capture the evolving structural properties of dynamic graphs. In contrast to previous approaches, we interactively apply an unsupervised graph learning method (graph embeddings) on a multiscale temporal hierarchy to directly learn structural properties. We furthermore use graph embeddings as a familiar representation for an analytical user task (e.g., similarity search, comparison) and utilize the visual exploration of different visual metaphors.

\subsection{Design Goals}

We derived three design goals for our visual design from the previously described problem description, the research gap, and also research challenges outlined in related work [6, 12, 13].

G1: Time-oriented visual analysis The visual analysis of dynamic graphs lacks new paradigms to examine structural (static) and temporal properties simultaneously. First, the identification of structural properties, e.g., clusters in a static graph, allows searching for similar structural properties over time. Such an exploration enables users to identify temporal states and continue to search for similar trends, reoccurring structures, and outlier structures.

G2: Temporal multiscale overview Our core idea is to provide an overview of multiple levels of temporal granularity, which facilitates users to relate higher-level overviews with low-level details. Such a multiscale overview allows detecting useful temporal analysis scales and gives additional context while navigating the temporal dimension (e.g., temporal filtering). For instance, a multiscale overview allows comparing states and transitions across multiple temporal granularities.

G3: Multiple visual metaphors The combination of different visual metaphors in a consistent interface allows adjusting the visualization to the data characteristics of particular intervals. It also increases the task coverage by enabling an analyst to adapt the visual representations to the task at hand. For example, matrix-based visualization techniques are better suited for dense subsequences of dynamic graphs.

\section{Multiscale Snapshots}

Multiscale Snapshots provides an overview of higher-level and finegrained temporal intervals of large-scale dynamic graphs. The approach reduces the complexity of the data by integrating temporal summarization and graph embeddings in an interactive multiscale visualization.

Our proposed visual analytics approach consists of three adjustable steps (see Fig. 1) to promote the exploration and summarization tasks for dynamic graphs [20]. The first step transforms the temporal dimension into a hierarchy of snapshots summarizing subsets of the dynamic graph into overlapping multiscale intervals. The second step reduces the complexity of the snapshots by embedding the summaries of the evolving topology into vector representations (e.g., using graph2vec [50]). The mapping of intervals into vector representation allows us to automatically adjust the visualization to highlight temporal states, trends, and outliers. The third step transforms the abstracted temporal data into a flexible and interactive hierarchical visualization and supports essential interaction as well as navigation methods to analyze the evolving graph structure visually. Furthermore, the visualization intends to increase the task coverage by combining different visualizations of dynamic graphs in a consistent interface. The following subsections describe each transformation step in more detail.

\subsection{Temporal Hierarchical Snapshots}

Dynamic graphs model relationships over time (e.g., social networks) and can be described as a number of $T$ static graphs $D G=$ $\left(G_{1}, G_{2}, \ldots, G_{T}\right)$. The temporal abstraction of dynamic graphs (e.g., aggregation, filtering) helps to reduce the data size, speed up temporal queries, support interactive analysis, and eliminates noise [47]. However, the temporal abstraction of sequence graphs into summaries remains challenging due to the selection of time granularity, which depends on many factors (e.g., application, data size), as well as the choice of abstraction method (e.g., summarization).

In various dynamic graph visualizations, a simple selection of one time granularity (uniform time-slicing) is used due to the simplicity of the approach [63]. In contrast, we propose a recursive temporal abstraction into a hierarchy with temporal overlaps to model multiple time granularities (see Fig. 2). We generate and stack multiple partitionings using uniform intervals (time slices) of different temporal granularities. We organize the stacked partitionings in a hierarchy that orders the different levels of abstraction (discretizations) from coarse to fine-grained temporal representations. Our bottom-up approach groups per default the temporal dimension into intervals of length $2^{l}$ with the level $l \in 1, \ldots,\lceil\log (T)\rceil$. Fig. 2 displays an example partitioning for a dynamic graph with eight time steps. The level one of the hierarchy 

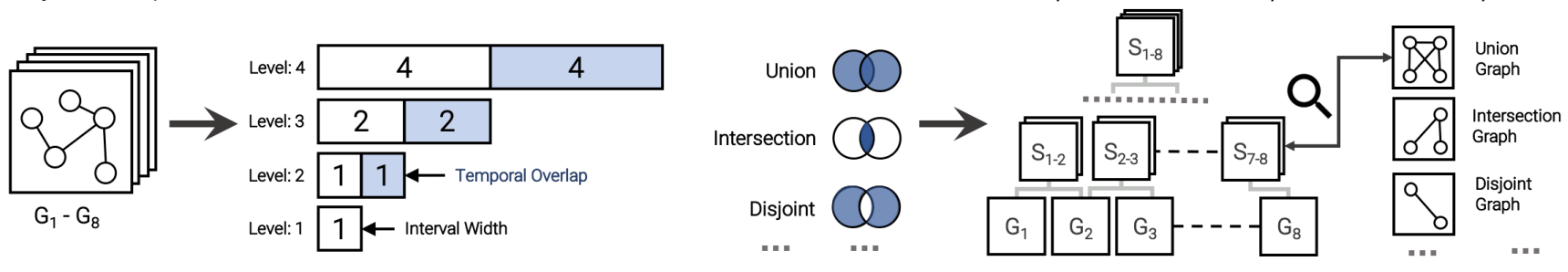

Fig. 2. The Figure displays the generation of temporal hierarchical snapshots for a dynamic graph with eight timesteps. First, the dynamic graph is partitioned into overlapping intervals at four levels of temporal granularity. The fourth level contains all data, and the first level consists of intervals of size one (static graphs). Second, abstraction methods are applied to the intervals to generate different compact summaries of the subsets of the dynamic graph. The result is a hierarchy of temporal snapshots that contains multiple summary graphs (e.g., union, intersection, and disjoint graph.

consists of intervals of length one, containing only one graph of the evolving data. The intervals are generated using a rolling window method, which facilitates time discretization without hard boundaries. The rolling window approach for level $l$ is computed by shifting the interval of width $2^{l}$ by the temporal overlap of width $2^{l-1}$. This results in each level having $\left\lceil T / 2^{l-1}\right\rceil$ intervals and the whole hierarchy having $(3 \cdot T)-1$ intervals. Essentially, as seen in Fig 2 each generated interval overlaps partly (e.g., per default half) with the next interval except for the level one (single graph) and the root node (all graphs). The default recursive partitioning into multiscale intervals results in the height of $\lceil\log (T)\rceil$. In practice, for most datasets, the height of the hierarchy is below 20 ( $<1$ million graphs). The width of time slicing can be modified to the application domain, for example, intervals with a width of a day, week, month, year.

The uniform time-slicing produces intervals of the same width for each level. The generation of non-uniform intervals for each level can be computed by applying temporal clustering techniques with varying input parameters. For example, the temporal clustering approach of Hadlack et al. [38] can be used to identify similar substructures based on graph properties to provide an overview of temporal trends. A hierarchy of temporal intervals can also be automatically generated by facilitating unsupervised learning with boundary detectors to obtain hierarchical temporal dependencies at different time scales [25]. The generation of such hierarchical temporal dependencies only works on time-series data of a dynamic graph, for example, on evolving graph metrics such as average clustering coefficient or density. Therefore, applying such methods remains challenging as there is no single graph metric that can capture all of the evolving graph structures.

The temporal abstraction aims to summarize and capture the evolving structural properties of sequences of graphs. We suggest utilizing multiple abstraction methods to generate diverse representations of the generated multiscale intervals as there is not a single abstraction method able to encode all evolving properties of a dynamic graph. We transform the intervals into graph summaries per default using set operations (union, intersection, disjoint graph). For example, the union operation abstracts the interval into a supergraph, which helps to provide an overview of all nodes and edges [37]. The three default graph summarization techniques (see Fig. 2) are the union graph that consists of a union of the set of all node and edge sets. The intersection graph which consists of all nodes appearing more than $i$-times in the interval. The disjoint graph consists of all nodes appearing less than $i$-times in the interval. We set the default value for the parameter $i$ to the interval overlap of an interval. If the values of $i$ are below the interval overlap, this will most probably result in successive intervals with a similar intersection and disjoint graphs.

We call the three computed graph summaries of an interval a snapshot $S_{l, k}$ (see Fig. 2). A snapshot aims to capture the structural and temporal properties of a sequence of graphs on level $l$ and the $k$ generated interval. The resulting intervals of the snapshots can be indexed in an interval tree to support the efficient support window queries, for example, identifying the best fitting interval to a user-defined period. We suggest, furthermore, utilizing more graph summarization methods based on the analytical task, data characteristics, and application domain. For example, we implemented a community detection algorithm (e.g., the Clauset-Newman-Moore algorithm [26]) to reduce the overall number of nodes in each static graph and to extract higher-level properties (e.g., meta-nodes and edges). For more graph summarization methods that can be added to our approach, we refer to the survey by Liu et al. [47].

Overall, the first step results in a hierarchy of abstracted snapshots at different temporal granularities (see Fig. 2). Every interval in the hierarchy contains multiple graph summaries, which can be used for different types of queries later on. For example, we can search for similar changes between intervals by using the disjoint graphs to identify reoccurring changes in the dynamic graph. The resulting temporal hierarchy of the dynamic graphs is used in the next step of our Multiscale Snapshots approach by mapping each summary to its vector representation.

\subsection{Multiscale Dynamic Graph Index}

As for the next step, the resulting hierarchical snapshots are learned and embedded into low-dimensional space to reduce the complexity of the graphs and speed up analytical tasks (e.g., similarity search). The main goal is to use unsupervised learning methods to model the similarities between the different multiscale temporal summaries and reduce the complex data characteristics to low-dimensional vectors preserving information. We apply a graph embedding (e.g., graph2vec [50]) to map all snapshot graphs (e.g., union and disjoint graphs) to vector representations. In contrast to earlier approaches (e.g., Van den Elzen [61]), unsupervised graph learning methods learn the topological structures of graphs and do not require any hand-engineered features. The embeddings can be precomputed and are also typically small enough to fit into main memory. To the best of our knowledge, Multiscale Snapshots is the first visual analytics approach to propose using unsupervised graph learning methods with different temporal granularities to visually analyze intervals sharing similar properties over time.

Recently, new unsupervised graph learning methods have been proposed to learn node and graph embeddings [66]. However, many of these methods mainly focus on learning static graph embeddings and cannot model the evolving properties of dynamic graphs [66]. In contrast to earlier approaches, we propose to model dynamic graphs by embedding summaries of subsets of the evolving data to capture the temporal dependencies between graphs. The analyst can apply graph embeddings such as graph2vec [50], GL2Vec [24], and FGSD [62] to the snapshots. The approach embeds all snapshots of the temporal hierarchy except for the level one (single graphs), which results in the embedding of $2 T-1$ snapshots. The resulting $2 T-1$ embeddings are also indexed to support efficient K-nearest neighbor search queries. We employ the following two index structures: an interval tree to support efficient temporal queries for the intervals, and an individual index structure for each level. We utilize for the indexing of the graph embeddings the proposed method of Malkov et al. [48] to perform a fast K-nearest neighbor search in each level.

In our evaluation (see Sec. 57, we compare different unsupervised graph embeddings, discuss the scalability of the approaches, and show that the embeddings of the snapshots are able to capture structural as well as temporal changes. 


\subsection{Multiscale Snapshots Visualization}

The final step applies a visual mapping to organize the temporal snapshots in a multiscale visualization to enable the visual analysis of the generated snapshots and uses the graph embeddings for analytical tasks. In the following, we describe the components of our visual and interaction design (see Fig. 3).

The visualization presents the hierarchy of snapshots and orders them from coarse to granular scale (top-down) and facilitates the horizontal (time) as well as vertical (time granularity) temporal navigation to search for similar properties over time (G1). The visualization stacks and displays the multiscale temporal abstractions (G2), allowing to analyze and compare the abstracted data at different temporal granularities. Presenting multiple abstraction levels enables us to gain more knowledge about the underlying abstracted dynamic graph (e.g., data distribution) [32]. The highest level (root) displays an aggregated version of the whole dynamic graph (e.g., union graph), and the bottom level enables us to depict a limited number of each time step. The levels in-between allow visualizing a subset of the generated snapshots in snapshots views (juxtaposed small multiples).

A snapshot view combines different visual metaphors in a consistent interface to increase the task coverage (G3) and displays one of the summary graphs (e.g., union graph). Every view enables users to depict the data using four kinds of visual metaphors (node-link, adjacency matrix, animation, and time series of graph metrics). We use these visual metaphors since the individual benefits, and drawbacks of the representations are well studied (e.g., graph layout and matrix reordering) [13]. We utilize multiple visual metaphors for certain intervals as the usefulness of dynamic graph visualization depends on the underlying changing data (e.g., sparse versus dense graphs) [22]. We consider our snapshot views as hybrid visualizations, as the view combines different visual metaphors in small multiple representations. Furthermore, the Clauset-Newman-Moore community detection algorithm [26] is applied to minimize visual clutter and to reduce the number of nodes in a snapshot view, if the size of the displayed summary graph exceeds a specific threshold (more than 100 nodes). This threshold is based on the size classification of Nobre et al. [52]. The resulting communities are then shown as meta-nodes and allow to filter the respective nodes and edges of the community for the entire Multiscale Snapshot visualization. For instance, the filtering of a structural cluster allows us to explore the evolving properties of the cluster in the displayed snapshot views. The snapshot view also visualizes derived structural properties using the background color of each snapshot view to highlight differences between adjacent visual metaphors. The derived properties (graph metrics) of the summary graph are used to identify and emphasize temporal or structural graph properties. For instance, we compute graph metrics such as the sum of the number of edges in a snapshot, which indicates the density of the underlying graph sequence. A linear color scale from light blue (low values) to darker blue (high values) is used to highlight changes of the derived structural properties [39].

The use of multiple levels of juxtaposed small multiples remains challenging due to limited display space and the preservation of the viewer's mental map. The simultaneous presentation of multiple levels and their snapshot views does not visually scale due to the restricted display space with an increasing number of snapshot views, as the readability of each view decreases. We, therefore, incorporate visual space abstraction methods to limit the number of displayed levels and snapshots views. The number of displayed levels is limited (default four), and during the vertical navigation, the respective lowest or highest level of temporal granularity is removed. Furthermore, we abstract snapshot views to reduce the number of shown visualizations and on particular snapshots while keeping the context of the abstracted views (focus-and-context principle). An abstracted snapshot is displayed as a compact colored rectangle without any visual representation. The background color can be mapped to extracted graph metrics of the selected summary graph, for example, the number of nodes as well as edges, average clustering coefficient, density, and transitivity. The coloring of such abstracted snapshot views enables the identification of intervals with specific properties, such as subsequences of dense graphs. In general, the usage of such color indicating graph properties allows

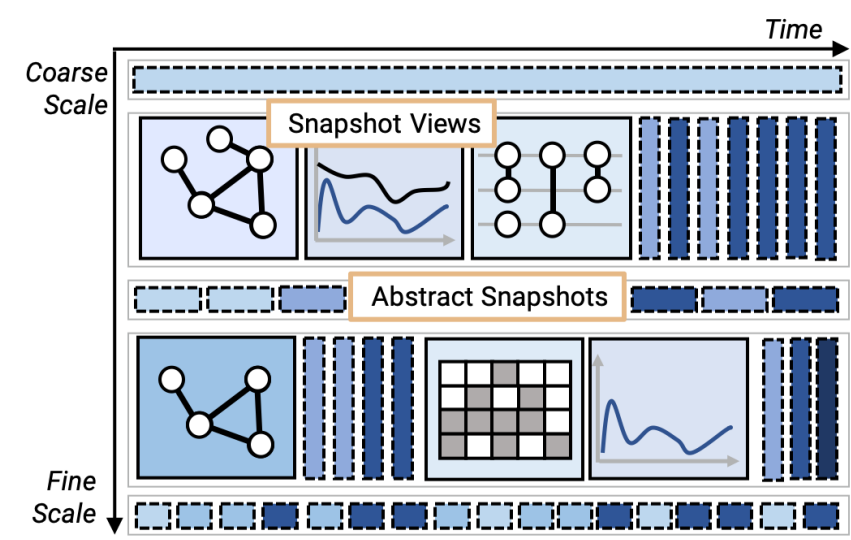

Fig. 3. The hierarchy organizes and displays the summaries from the snapshots from coarse to fine-grained representations. The visual metaphors in each snapshot view can be manually or semi-automatically adapted. The snapshot views can be abstracted to reduce the number of displayed views and duplicate information. The background color of each snapshot is mapped to graph metrics (e.g., number of edges).

users to identify and compare temporal intervals [59|. The abstraction can be done manually by reducing individual snapshot views or whole levels of the hierarchy, using a user-driven threshold, and an automated abstraction algorithm.

The automated algorithm limits the number of intervals by traversing the hierarchy and abstracting redundant information. The algorithm abstracts snapshots if the number of views exceeded a specific threshold, or if the algorithm detects duplicate displayed periods. The algorithm traverses each level of the hierarchy (top-down) and compares the displayed snapshots at each level against each other. If coarse snapshots (high level) are displayed in the fine-grained snapshots (low levels), they are abstracted. The automatic abstraction is done based on overlapping windows in the interval tree, which means that the snapshot view with the highest overlap with low-level snapshots is abstracted. The algorithm compares, for example, the time interval of the root view against all other not abstracted snapshots, and if the periods of these more granular levels display the majority of temporal information of the root view, then the root snapshot view is abstracted. The thresholds for the automatic abstraction algorithm, such as the overall number of levels and snapshot views, are adjustable by the analyst.

Furthermore, we aim to preserve the viewer's mental map, which increases the readability and interpretability of the evolving data [54]. To maintain the viewer's mental map, we fix and use one global layout for each visual metaphor. For instance, we compute one layout for the overall supergraph of the dynamic graph. Furthermore, the usage of linking and brushing aims to preserve the mental map between adjacent snapshots that are using different visual metaphors and the different levels of abstraction in the hierarchy.

Multiscale Snapshots utilizes the graph embeddings for automated analysis to identify trends, reoccurring, and outlier states. For example, an analyst can select a snapshot view and can apply a k-nearest neighbor search query to detect similar summary graphs (see the query interface Fig. 47. The detected k-nearest neighbor snapshots can also be disaggregated to more granular views using the interval tree (drilldown). The similarity search can also be applied to a particular type of summary graph, for instance, search for similar intersection graphs. Such similarity queries also enable to semi-automatically abstract and adapt the displayed snapshot views. The k-nearest neighbor queries can also be applied to particular intervals (subqueries) and to specific levels, which allows examining the summaries of the dynamic graphs in a top-down manner. The embeddings can also be used to cluster levels of the hierarchy and to identify outlier states by applying outlier detection algorithms [2].

In summary, the visual design provides an overview of snapshots of a dynamic graph by combining automatic analysis methods with visual space abstraction methods (focus-and-context). 


\subsection{Multiscale Snapshots Prototype}

We show the usefulness of our approach by applying it to real-world data using our prototype ${ }^{1}$ The prototype consists of two components (see Fig. 4 A-B): the Multiscale Snapshots visualization and the query interface. Both components allow users to explore and semiautomatically search for similar temporal states in the dynamic graph.

The Multiscale Snapshots visualization consists of a toolbar, the stacked snapshot views, and two context bars. The toolbar facilitates the application of automated analysis methods (e.g., open the query interface) and visualizes the summary graphs of the snapshots (e.g., display union or intersection graph). Furthermore, the toolbar enables changing the data space abstraction methods (e.g., filter and cluster nodes) and adapting visual transformations (e.g., reordering algorithms for matrix visualization). The prototype displays by default the root of the hierarchy as a supergraph (union graph) using a node-link diagram visualization. The layout of the node-link diagram is computed once for the root supergraph using per default the Fruchterman-Reingold [34] layout algorithm and later used for all snapshot views. The hierarchy enables an analyst to navigate horizontally (time) or vertically (overview to detail) on the temporal dimension. The two context bars display additional information during the horizontal and vertical navigation of the temporal dimension. The time context bar on the top shows the visualized intervals, and the level context bar on the right allows to add and remove levels. Each snapshot view can be visually analyzed via zooming, panning, brushing, and changing the layout in all views (e.g. reordering of the matrix) to make visual patterns more apparent [15] The visual transformations for individual or all snapshot views can be adjusted by the analyst to enable the adaption of visual metaphors to the underlying sequence of graphs, such as switching for periods of dense sequences of graphs to matrix visualization. The prototype also enables filtering by specific graph properties (e.g., node degree) and clustering [26] to reduce overall displayed elements to extract higher-level features (e.g., meta-nodes and edges). The background color of each snapshot view can be mapped to extracted graph metrics and computed node characteristics (e.g., clustering coefficient) to node size. To apply a k-nearest neighbor query, an analyst has to select a specific summary graph in a snapshot view.

The query interface allows applying specific k-nearest-neighbor queries to search for similar summary graphs on all or particular levels of temporal granularity. The query interface displays each time dimension of a level and encodes the currently visualized and already investigated snapshots via color. This additional information helps to keep an overview of the already explored snapshots of all levels. The timelines can be ordered by different features, such as by the percentage of explored snapshots. An analyst can select the levels, time interval, and the summary graph type (e.g., only union graphs) to apply the $\mathrm{k}$-nearest neighbor search. The number of k-nearest neighbors is also configurable. The query results are displayed as dots on the timeline, and the euclidean distance between the underlying graph embeddings is mapped to the opacity of the dot. The analyst has to select a subset of the nearest-neighbors, which are then displayed in the Multiscale Snapshots visualization. The selected results are shown as snapshot views and allow users to analyze and compare similar temporal states in lower or higher temporal granularities against each other.

\section{Evaluation}

In the following section, we evaluate the two main components of the Multiscale Snapshots approach. We provide a usage scenario to demonstrate how the visual analytics approach can be utilized to gain an overview of temporal summaries in a dynamic graph. We furthermore quantitatively evaluate the similarity ( $k$-nearest neighbors) search of the graph embeddings with synthetic and real-world datasets.

\subsection{Usage Scenario}

We demonstrate the applicability of our approach using a real-world, large-scale dynamic graph of the website Reddit [46]. Reddit is a social news aggregation website with 440 million active users who

\footnotetext{
https://github.com/eren-ck/MultiscaleSnapshots
}

can publish and upvote posts of interest (e.g., link to news sites) in particular communities (subreddits). The analyzed dataset is a dynamic hyperlink graph in which nodes are subreddits, and edges are hyperlinks posted between subreddits.

Tasks In the following, we outline the actions that a fictitious analyst takes to discover structural and temporal changes during the 2016 US presidential elections (see Fig. 4). A task in the visual analysis of such hyperlinks is to gain an overview of temporal events (e.g., political scandals), identify reoccurring links between communities, and examine structural changes within groups of subreddits. The visual analysis of such data with state-of-the-art visual analytics approaches remains challenging due to the varying duration of such events. For example, the length of political scandals varies significantly due to media exposure and their temporal context (e.g., during elections). In contrast to previous approaches, Multiscale Snapshots enables us to detect events/states of different temporal lengths due to the temporal multiscale modeling.

Dynamic Graph The Reddit dataset [46] comprises hyperlinks between subreddits from the 1st January 2016 to 30th November 2016. The resulting dynamic graph contains 7974 graphs (grouped by hours), 18546 subreddits (nodes), and 88328 hyperlinks between subreddits (edges). The timestamped hyperlink posts have a sentiment label indicating if the post is positive or negative towards the other subreddit. The dynamic graph index was computed using the Graph2Vec [50] embedding approach for 80 epochs, and three summary graphs for each snapshot were generated (union, intersection, and disjoint graphs). The validation of the detected findings is done by comparison to the ground truth of real historical news coverage.

Initial Setup Per default, our prototype displays the entire graph as an aggregated node-link diagram (supergraph). Then, based on the Kamada-Kawai algorithm [43], a global layout is computed for all snapshot views once. This way, the mental map is preserved during the visual analysis. Furthermore, snapshot views that display more than 100 nodes are automatically clustered using the greedy Clauset-Newman-Moore community detection algorithm [26] to reduce the number of nodes and to extract higher-level properties (e.g., meta-nodes and meta-edges). The clustering of the approximately 8000 nodes of the analyzed data reveals several clusters of subreddits (e.g., computer games subreddits).

Starting Point: Election Week First, the analyst wants to analyze the election week of the 2016 US presidential race (8th November 2016) to identify important groups of political subreddits. The analyst enters the dates of the election week, and the prototype automatically searches for the best fitting snapshot period using the interval tree. The prototype depicts a union graph of election week, and the analyst maps the size of the cluster to the node size to discover large groups of subreddits (see Fig. 4.A). He selects the largest visible meta-node and all underlying political subreddits of the cluster. He filters these political subreddits as he assumes that the political subreddits of the election week have also been active in the political discourse of the whole election.

Similarity Search To identify political events similar to the election week in the dynamic graph, the analyst searches for similar embeddings using the election weeks supergraph. Using the query interface (see Fig. 4.B), he queries the five nearest neighbors for each level and sorts the levels by the similarity of the embeddings. The executed nearest neighbor query is calculated on the unfiltered summary graphs for each snapshot, which means that the similarity search results will include false-positives that do not necessarily include any political subreddits. The analyst discovers that the results of the query are similar embeddings on the second (2-hour periods) and third level (4hour periods), which means that these rather short sequences of graphs consist of a subset of hyperlinks similar to the ones during the election week. The analyst selects the three closest neighbors for both levels and therefore navigates from a high temporal aggregation (a week) to a lower granularity (2-4 hours). Three queried snapshots are empty, meaning the views do not contain any of the previously filtered political subreddits. The empty views are presumably false positives that capture other graph sub-structures of the election week. The analyst removes 


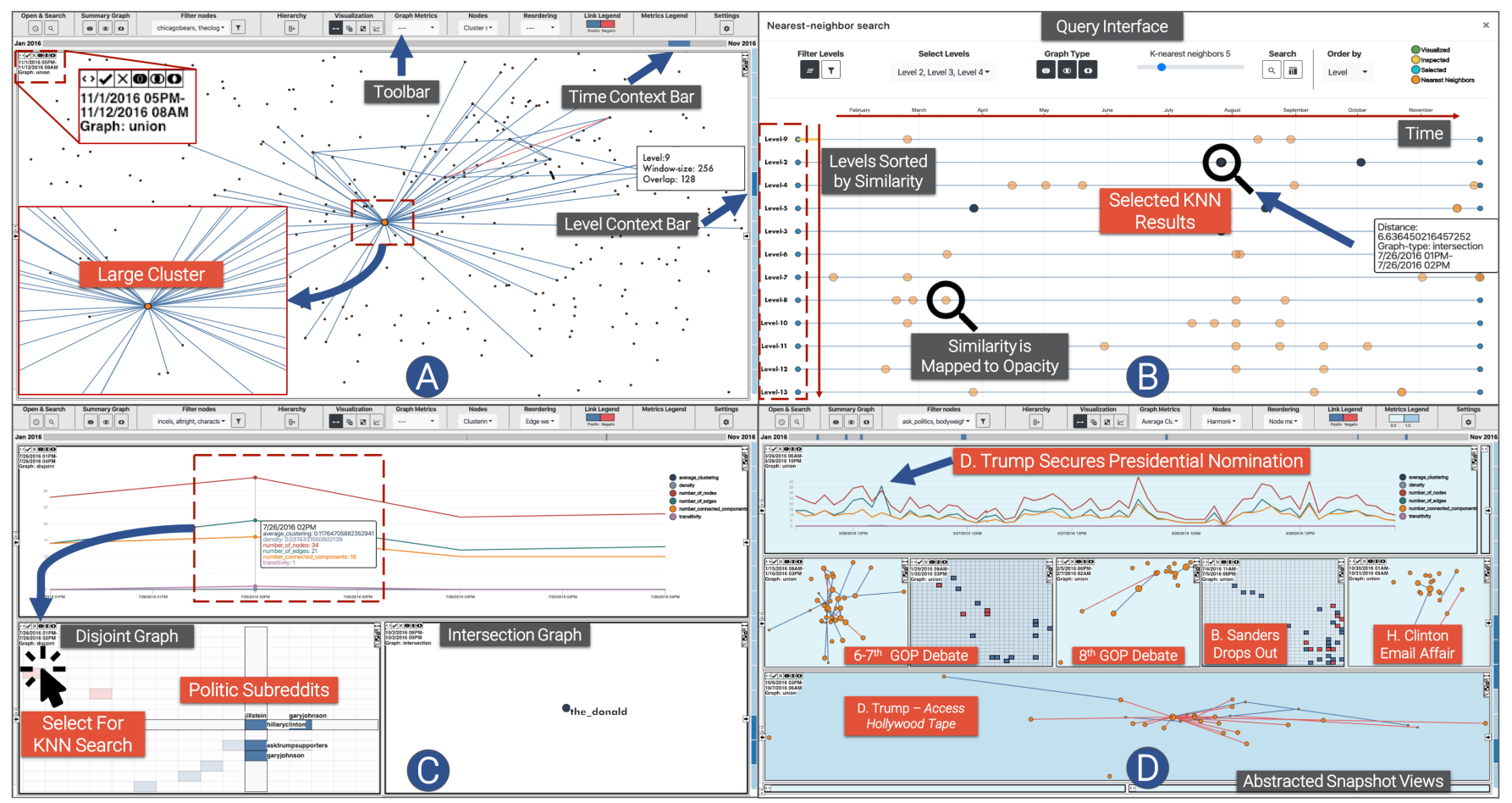

Fig. 4. The prototype implementation consists of two primary components the Multiscale Snapshots visualization (A) and the query interface (B). The figures present the visual analysis of the Reddit hyperlink dataset (see Sec. 5.1. The displayed nodes are subreddits, and the edges are timestamped hyperlinks between subreddits with either positive (blue) or negative (red) sentiment. The displayed nodes are subreddits, and the edges are timestamped hyperlinks between subreddits with either positive (blue) or negative (red) sentiment. The example illustrates by the case of the 2016 US election how the approach allows searching for similar temporal states in the dynamic graph. The intermediate steps of the visual analysis and the resulting interfaces are presented in the sub-figures C-D. In D, the results of the visual analysis by similarity search are displayed, which are significant events in the timeline of the presidential election.

the three empty snapshots and examines the remaining three snapshots by changing the visual mapping from a node-link diagram to the time series of graph metrics.

Fine-Grained Temporal Analysis The three remaining snapshots contain a different amount of nodes. The intersection graph on the second level contains only one subreddit (the_donald), which means the subreddit was referenced in both graphs of the snapshot ( 2 hours). The analyst discovers that a high-level summary graph (disjoint) includes a displayed snapshot of the second level. The unexpected overlap steers the analysts towards the low-level disjoint graph, which seems to also be the peak in the time series of graph metrics. The time series presents the number of nodes, edges, as well as connected graph components, the graph density, the average clustering coefficient, and the transitivity over time. It seems that this second level snapshot is essential for the results of the search as the snapshot has similar graph structures compared to the supergraph of the election week. The peak is presented as a matrix visualization (see Fig. 4.C) and can be attributed to the events of the national democratic convention where $\mathrm{H}$. Clinton was nominated for the presidential election. The disjoint graph represented as a matrix visualization (see Fig. 4. C) can be associated to the political event of the democratic nomination $\mathrm{H}$. Clinton which resulted in a cluster of hyperlinks between political subreddits (e.g., hillaryclinton, asktrumpsupporters, and garyjohnson) and other hyperlinks between political subreddits (e.g., communism101, altright, and crazyideas) The analyst uses the snapshot (disjoint graph) for another similarity search. He expects the similarity search to return more political events because the low-level graph embedding of the two-hour snapshot contains mainly linked political subreddits.

Searching for Political Events The similarity search finds many similar snapshots at different temporal granularities, which indicates that these political events also seem to be discussed for different periods. The query returns several similar snapshots of the sixth level with an interval length of 32 hours, which can refer to potential political events and their daily news coverage scheme (see Fig. 4.D). The analyst investigates the different snapshot views, mostly union, and disjoint graphs, and abstracts all snapshot views with only a few subreddits. The remaining presented snapshots are on levels 5-7 and contain intervals of 16, 32, and 64 hours. The analyst maps the average clustering coefficient to the background color of each snapshot view to identify periods with structural clusters. He changes the visual metaphors of the dense snapshots to matrix visualizations and the higher-level periods to the time-series metaphor. The different visual metaphors allow the analyst to put the events on lower levels into the overall temporal context, for example, the analyst can relate how the linkage behavior between subreddits declines after political scandals.

Political Events and Scandals The analyst then visually analyzes the periods and sees that during the selected periods political subreddits link each other, mainly in a positive (blue edge) or negative (red edge) way. Various subreddits such as the_donald and asktrumpsupporters usually have positive hyperlinks between each other. He examines external resources of the timeline of major events for the 2016 US elections and can refer the presented snapshot views to events in the presidential race. The sixth level of the hierarchy displays several GOP (republican party) political debates, B. Sanders dropping out of the primary election, and the $\mathrm{H}$. Clinton Email affair. The analyst is also able to identify structural changes between the views, for instance, after B. Sanders drops the linking activity of some subreddits (e.g., SandersForPresident or Democratic Socialism) declines. The snapshot view of 6-7th October on the fifth level stands out as it mostly contains negative links between the subreddits. The analyst can relate the period to the leaked tapes of the 2005 Access Hollywood show in which D. Trump brags about sexual exploits and also on the same day WikiLeaks published the email of $\mathrm{H}$. Clintons campaign manager revealing her paid Wall Street speeches. The analyst wants to analyze this snapshot further and displays the supergraph as an animated node-link diagram to examine the spread of news between the subreddits on an hourly basis. 
During the further analysis of snapshot views, the analyst can also detect other events, for instance, the final nomination of D. Trump by the GOP, which results in visible changes in the time series plot of graph metrics. He also detects some events, which he cannot relate to actual major political events directly. Those events are probably general political discussions initiated by Reddit users or targeted distribution of news from public-relations groups or political bots. To further investigate such events, the analyst can select these non-assignable events and search for similar periods, for example, to identify the reoccurring post of political bots.

\subsection{Experimental Evaluation}

The generated graph embeddings for the multiscale snapshots are independent of any analytical task and can be used for clustering, graph prediction, and outlier detection. In the following, we show that the multiscale graph embeddings allow us to search for similar sequences of graphs. Across all experiments, we use the same parameter settings for the generation of the multiscale index.

Problem Background A similarity search for a set of graphs can be interpreted as a query to return $k$-nearest neighbors to a specific graph. An exhaustive simple brute-force algorithm would compute the distance between all graphs, for example, the graph editing distance (GED) |21] and return the list of $k$ nearest graphs. However, the extensive brute-force approach does not scale as the GED computation is not feasible for graphs with more than 16 nodes [18]. Therefore, heuristics are usually applied to decrease the computation effort of $k$ nearest neighbor queries, which in turn frequently reduces the accuracy of the results. In the following, we apply window queries for sequences of graphs to show that summarization methods (e.g., union graph) can capture some temporal characteristics.

Datasets We evaluate the performance of similarity search on synthetic and real-world data. We generated five synthetic dynamic graphs using the dynamic stochastic block model with diminishing communities [35]. The synthetic datasets consist of 150 nodes with three communities and 100-time steps, containing varying amounts of diminishing communities (up to 20-time steps) in which two nodes are exchanged for each time step. We evaluate the approach with realworld datasets. The Reddit data [46] is a dynamic hyperlink graph with subreddits (nodes) and hyperlinks or crossposts between subreddits (edges). The Wikipedia dataset [53] consists of a dynamic graph that captures the editing behavior (edge) between Wikipedia Talk pages (nodes). For each real-world dataset, we preprocess the data by computing a supergraph for each hour, which generates descriptive dynamic graphs with more than two nodes per time step. We evaluated our approach on randomly picked subsets (100 graphs) of the real-world data. We select a subset of the data as the computation of the following ground truth is quite expensive.

Ground Truth We calculate a ground-truth similarity score for the $k$-nearest neighbor search by computing the distance between the input and all other graphs. We employ the following similarity measure between two graphs. Our similarity measurement first models the graphs as two adjacency matrices $A$ and $B$ and then compute for each matrix the singular values via the singular value decomposition. Afterward, we calculate the fnorm using

$$
\text { fnorm }=\sqrt{\sum_{i=0}^{S} \sigma_{i}^{2}}
$$

We define the distance between two graphs as

$$
\operatorname{madist}(A, B)=|\operatorname{fnorm}(A)-\operatorname{fnorm}(B)|
$$

Using the given similarity measurement, we compute the distances between all graphs to obtain a ground-truth of $k$-nearest neighbors.

Baseline Methods We used three unsupervised graph learning methods on the described datasets. The graph embeddings are applied once with and once without the multiscale temporal modeling. We used the following graph embedding methods [55] with the described input parameters:
- graph2vec [50]: 250 epochs, 0.025 learning rate, 2 WeisfeilerLehman iterations, and 128 dimensions.

- GL2Vec [24]: 250 epochs, 0.025 learning rate, and 128 dimensions.

- FGSD |62|: 200 number of histogram bins with a the histogram range of 20 .

For window queries for the single graph embeddings without any summarization methods, we utilize the median value of the embeddings as the representative value of the interval. We use the median as the average of the embeddings as these can lead to potential distortions in the embedded space. For the multiscale temporal embedding, we applied only one temporal summarization method to generate a union graph for each snapshot. The searched intervals for the $k$ nearest neighbor search are extracted before training of embedding techniques. We randomly extracted five intervals with different lengths $(1-8)$ from the dynamic graph and randomly removed one node from each graph.

Evaluation Metrics The following metrics are used to evaluate the approach. We compute the accuracy of the 5-nearest neighbor queries based on the ground-truth. For the accuracy computation, we do not incorporate the ordering of the nearest neighbors and expect only the presence in the result set.

Experimental Setup All experiments were computed on a computer with two CPU cores (Intel i7-6567U 3.30GHz) and 16 GB RAM. The experiment was repeated five times, and the average accuracy was computed for each randomly picked interval with different lengths.

Results The results are described in Table 2 The results indicate that FGSD [62] works best to identify nearest neighbors on an embedding basis using the median. The results show that the single graph embeddings have a higher accuracy on the synthetic data. In contrast, the real-world datasets indicate different results by demonstrating equal or improved results by using the multiscale index for longer intervals $(<4)$. An explanation for this can be the fact that there is a drastic difference between the topology of the synthetic and real-world datasets. For example, in the real-world data nodes and edges are added and removed more frequently between time steps. The synthetically generated dataset has a quite high density, while in contrast to this, the real-world datasets are much more sparse. For example, in the synthetic data, the nodes are just moved between the clusters, so only edges change over time. These synthetic properties prevent supergraphs from encoding the topological changes over time. Therefore, the multiscale graph index requires a different temporal summarization method to capture the changes of the synthetic dataset (e.g., disjoint graph).

\section{Discussion}

The Multiscale Snapshots approach consists of three steps: (1) applying temporal summarization methods, (2) utilizing graph embedding methods to reduce the size of the generated graph summaries, and (3) the visual analysis of the generated snapshots. Our quantitative evaluation indicates the usefulness of the multiscale graph embeddings, and the usage scenario shows the application of the approach to real-world data. Overall the utility of the approach yet depends on multiple aspects (e.g., summarization method and graph embedding), the data characteristics (e.g., data distribution), and the task at hand (e.g., outlier analysis).

Steps (1-2) involve multiple methods with parameters. For instance, the graph embeddings methods require a definition of the number of layers and epochs. For an analyst, such parameter choices pose a challenge as he has to determine suitable methods and their input parameters to generate useful embeddings. We consider the flexibility of using different temporal summarization methods and graph embeddings as an advantage of our approach and a possibility for future work.

Another challenge for steps (1-2) is the computational scalability for the precomputation of the embeddings. For example, the computation of a dynamic graph of length $T$ with $|V|$ nodes and $|E|$ edges require for only union graphs $O(\log (T) \cdot(|V|+|E|))$ memory and time complexity. We speed up the computation of temporal summaries by parallelizing each levels snapshot generation and the usage of an interval tree. Goyal and Ferrara [36] surveyed the time complexity of graph embeddings, 
(C) 2020 IEEE. This is the author's version of the article that has been published in IEEE Transactions on Visualization and Computer Graphics. The final version of this record is available at: $x x . x x x x / T V C G .201 x . x x x x x x x /$

\begin{tabular}{|r|c|c|c|c|c|c|c|c|c|c|c|c|c|c|c|}
\hline & \multicolumn{4}{|c|}{ Synthetic Data } & \multicolumn{4}{|c|}{ Reddit Data } & \multicolumn{4}{|c|}{ Whikipedia Data } & 53] \\
\hline \hline Interval length & 1 & 2 & 3 & 4 & 8 & 1 & 2 & 3 & 4 & 8 & 1 & 2 & 3 & 4 & 8 \\
\hline \hline Graph2Vec & 0.096 & 0.146 & 0.096 & 0.15 & 0.096 & 0.332 & 0.066 & 0 & 0 & 0.264 & 0 & 0 & 0 & 0 & 0.066 \\
\hline GL2Vec & 0.096 & 0.122 & 0.024 & 0.024 & 0 & 0.198 & 0.132 & $\mathbf{0 . 2 6 6}$ & 0.066 & 0.066 & 0.066 & 0 & 0.066 & 0 & 0.066 \\
\hline FGSD & 0.146 & $\mathbf{0 . 2 2 4}$ & $\mathbf{0 . 1 9 8}$ & $\mathbf{0 . 1 9 8}$ & 0.048 & $\mathbf{0 . 4 6 4}$ & $\mathbf{0 . 3 3 2}$ & 0 & $\mathbf{0 . 1 3 2}$ & 0.066 & $\mathbf{0 . 8 6 6}$ & $\mathbf{0 . 1 3 2}$ & 0.066 & 0.132 & 0.134 \\
\hline Multiscale Graph2Vec & $\mathbf{0 . 1 4 8}$ & 0.072 & 0.096 & 0.072 & $\mathbf{0 . 1 2 2}$ & 0.266 & 0.264 & 0 & 0.066 & 0.264 & 0.234 & 0.066 & 0.066 & 0.066 & 0.198 \\
\hline Multiscale GL2Vec & $\mathbf{0 . 1 4 8}$ & 0.072 & 0.096 & 0.072 & $\mathbf{0 . 1 2 2}$ & 0.198 & 0.132 & 0.066 & $\mathbf{0 . 1 3 2}$ & $\mathbf{0 . 4}$ & 0.234 & 0.066 & 0.198 & 0.132 & 0.198 \\
\hline Multiscale FGSD & 0.146 & 0.096 & 0.096 & 0.072 & $\mathbf{0 . 1 2 2}$ & 0.264 & 0.264 & 0 & 0 & 0.332 & 0.466 & 0.066 & $\mathbf{0 . 2}$ & $\mathbf{0 . 1 9 8}$ & $\mathbf{0 . 2 6 6}$ \\
\hline
\end{tabular}

Table 2. The Table presents the quantitative evaluation results of the k-nearest neighbor search with and without the multiscale graph index for different graph embedding methods. The average accuracy values for window queries of different lengths (1-8) are depicted for each dataset. The experiment was repeated five times on synthetically generated data and with randomly selected subsets of real-world data. The results of the evaluation indicate an improved accuracy on window queries on the listed real-world dataset.

and scalable embeddings run in the time complexity of $O(|E|)$. Due to the time and memory complexities, we suggest computing the graph embeddings for large scale dynamic graphs on a server.

Step (3) aims to display the temporal dimension at multiple scales, which poses new user-related aesthetic challenges [12]. To preserve the mental map, we compute and use only one layout for each applied visual metaphor (e.g., global node-link diagram layout). An analyst can change the global layout for all snapshot views, for instance, by reordering the cells and rows of the adjacency matrix visualization. The snapshot views can also result in adjacent snapshots that display different dynamic graph visualization (e.g., node-link and matrix visualization). Consequently, the mental map between such views cannot be preserved as it is not possible to track and identify changes efficiently. We provide brushing and linking methods to minimize the cognitive load of identifying nodes in different visual metaphors. Another limitation of our approach is the fact that specific snapshots can be mistaken for other periods (temporal aliases [12]). We aim to overcome such temporal aliases by displaying the period in each snapshot view and the time context bar highlighting the underlying period in the overall temporal context. We consider these aesthetic challenges [12] as open possibilities for the development of new methods for the interactive comparison of two or more snapshots at different granularities. For example, the investigation of how such mixed visual metaphors impact the overall user experiences poses an opportunity for future work.

The applied methods during the visual analysis influence the computational and visual scalability of our approach. For instance, the live computation of displayed graph summaries scales linearly to the number of time steps and the size of the evolving graphs. Furthermore, the real-time analysis of snapshots can suffer based on the algorithmic time complexities of applied methods, for example, the Clauset-NewmanMoore community detection algorithm [26]. A possible solution to these challenges is to investigate how graph embeddings can be utilized to guide an analyst towards temporal changes to speed up the analysis process. Furthermore, the display space limits the visual scalability and readability of structural properties in a snapshot view since they depend on the number of presented snapshots. To address this, we limit and automatically abstract the number of depicted snapshot views to provide visually readable representations. The limit for the number of snapshots is adjustable and is as a heuristic limited to six snapshot views for each level. The visual scalability can also be increased by adapting the visual metaphors based on graph properties, such as, automatically presenting matrix-based visualization for dense graphs.

We showed the applicability of the approach through the visual analysis of similar periods in a dynamic hyperlink graph, which required an initial starting point for the similarity search (e.g., the election week). An analyst has to be aware of such states in advance or apply automated analysis methods to identify them, for instance, by using change-point detection [5] algorithms on the graph embeddings. Furthermore, the variety of functionality also affects the usability of the approach since the application prototype can be challenging to use for untrained users. In general, the usage of user guidance in combination with the potential application of more automatic analysis methods (e.g., outlier detection algorithms), can help to set high-level snapshots in the context of lowlevel snapshots, drill down the temporal hierarchy, and steer the user towards a useful combination of data and visual transformations to highlight specific trends. For example, the utilization of sub-queries in the temporal hierarchy can be used to steer an analyst towards fine-grained states with particular graph properties (e.g., motifs).

A limitation of our work is the lack of a formal comparative study to compare Multiscale Snapshots with other visual analytics approaches. In general, such a comparison remains challenging as our approach allows us to integrate visualization techniques (e.g., van Elzen et al. [61]), which is a simple way to increase the overall task coverage. Despite the shortage of a comparative study, our quantitative evaluation and the usage scenario highlight key benefits of our approach, such as the multiscale embedding of sequences of graphs to speeds up analytical tasks (e.g., similarity search). Graph embeddings come with the sacrifice of information loss compared to methods such as the computation of graph editing distance (GED) [21]. In future work, we aim to overcome shortcomings by integrating new visual metaphors to allow analysts to examine snapshots and their graph embeddings to understand and interpret the quality of the underlying graph embeddings.

\section{Conclusion}

In this paper, we presented Multiscale Snapshots, a visual analytics approach, to provide an overview of a dynamic graph. The approach consists of three steps: creating multiscale temporal summaries, applying graph embeddings, and the semi-automatic visual analysis. The combination of the steps enables us to visually explore how temporal and structural properties affect the overall dynamic graph. We implemented a prototype and showed in a quantitative evaluation that the approach helps to identify similar temporal states in artificial and real-world dynamic graphs. We also show the applicability by a usage scenario analyzing a real-world dataset, demonstrating that patterns in dynamic graphs can be visually analyzed over time.

The application of Multiscale Snapshots and the underlying multiscale temporal analysis paradigm is not limited to dynamic graphs and can be extended in several ways to work with any temporal data. For instance, the Multiscale Snapshots approach can be adjusted to support the user-driven analysis of multivariate time-series data.

\section{ACKNOWLEDGMENTS}

This work was partly funded by the Deutsche Forschungsgemeinschaft (DFG, German Research Foundation) under Germany's Excellence Strategy - EXC 2117 - 422037984 and the European Unions Horizon 2020 research and innovation programme under grant agreement No 830892 .

\section{REFERENCES}

[1] J. Abello and F. Van Ham. Matrix zoom: A visual interface to semiexternal graphs. In IEEE symposium on information visualization, pp. 183-190. IEEE, 2004.

[2] C. C. Aggarwal. Outlier analysis. In Data mining, pp. 237-263. Springer, 2015.

[3] J.-w. Ahn, M. Taieb-Maimon, A. Sopan, C. Plaisant, and B. Shneiderman. Temporal visualization of social network dynamics: Prototypes for nation of neighbors. In International Conference on Social Computing, Behavioral-Cultural Modeling, and Prediction, pp. 309-316. Springer, 2011.

[4] W. Aigner, S. Miksch, W. Müller, H. Schumann, and C. Tominski. Visual methods for analyzing time-oriented data. IEEE transactions on visualization and computer graphics, 14(1):47-60, 2008. 
[5] L. Akoglu and C. Faloutsos. Event detection in time series of mobile communication graphs. In Army science conference, vol. 1, 2010.

[6] D. Archambault, J. Abello, J. Kennedy, S. Kobourov, K.-L. Ma, S. Miksch, C. Muelder, and A. C. Telea. Temporal multivariate networks. In Multivariate Network Visualization, pp. 151-174. Springer, 2014.

[7] D. Archambault, T. Munzner, and D. Auber. Grouseflocks: Steerable exploration of graph hierarchy space. IEEE transactions on visualization and computer graphics, 14(4):900-913, 2008.

[8] D. Archambault, H. Purchase, and B. Pinaud. Animation, small multiples, and the effect of mental map preservation in dynamic graphs. IEEE Transactions on Visualization and Computer Graphics, 17(4):539-552, 2011.

[9] B. Bach, N. Henry-Riche, T. Dwyer, T. Madhyastha, J.-D. Fekete, and T. Grabowski. Small multipiles: Piling time to explore temporal patterns in dynamic networks. In Computer Graphics Forum, vol. 34, pp. 31-40. Wiley Online Library, 2015.

[10] B. Bach, E. Pietriga, and J.-D. Fekete. Visualizing dynamic networks with matrix cubes. In Proceedings of the SIGCHI conference on Human Factors in Computing Systems, pp. 877-886. ACM, 2014.

[11] B. Bach, C. Shi, N. Heulot, T. Madhyastha, T. Grabowski, and P. Dragicevic. Time curves: Folding time to visualize patterns of temporal evolution in data. IEEE transactions on visualization and computer graphics, 22:559-568, 2015.

[12] F. Beck, M. Burch, and S. Diehl. Towards an aesthetic dimensions framework for dynamic graph visualisations. In 2009 13th International Conference Information Visualisation, pp. 592-597. IEEE, 2009.

[13] F. Beck, M. Burch, S. Diehl, and D. Weiskopf. A taxonomy and survey of dynamic graph visualization. In Computer Graphics Forum, vol. 36, pp. 133-159. Wiley Online Library, 2017.

[14] B. B. Bederson, L. Stead, and J. D. Hollan. Pad++: Advances in multiscale interfaces. In Conference on Human Factors in Computing Systems: Conference companion on Human factors in computing systems, vol. 24, pp. 315-316, 1994.

[15] M. Behrisch, B. Bach, N. Henry Riche, T. Schreck, and J.-D. Fekete. Matrix reordering methods for table and network visualization. In Computer Graphics Forum, vol. 35, pp. 693-716. Wiley Online Library, 2016.

[16] S. Bender-deMoll and D. A. McFarland. The art and science of dynamic network visualization. Journal of Social Structure, 7(2):1-38, 2006.

[17] A. Bezerianos, F. Chevalier, P. Dragicevic, N. Elmqvist, and J.-D. Fekete. Graphdice: A system for exploring multivariate social networks. In Computer Graphics Forum, vol. 29, pp. 863-872. Wiley Online Library, 2010.

[18] D. B. Blumenthal and J. Gamper. On the exact computation of the graph edit distance. Pattern Recognition Letters, 2018.

[19] U. Brandes and D. Wagner. Analysis and visualization of social networks. In Graph drawing software, pp. 321-340. Springer, 2004.

[20] M. Brehmer and T. Munzner. A multi-level typology of abstract visualization tasks. IEEE transactions on visualization and computer graphics, 19(12):2376-2385, 2013.

[21] H. Bunke. What is the distance between graphs. Bulletin of the EATCS, 20:35-39, 1983.

[22] M. Burch and T. Reinhardt. Dynamic graph visualization on different temporal granularities. In 201721 st International Conference Information Visualisation (IV), pp. 230-235. IEEE, 2017.

[23] M. Burch and D. Weiskopf. Flip-book visualization of dynamic graphs. Int. J. Software and Informatics, 9(1):3-21, 2015.

[24] H. Chen and H. Koga. Gl2vec: Graph embedding enriched by line graphs with edge features. In International Conference on Neural Information Processing, pp. 3-14. Springer, 2019.

[25] J. Chung, S. Ahn, and Y. Bengio. Hierarchical multiscale recurrent neural networks. In 5th International Conference on Learning Representations, ICLR 2017, 2019.

[26] A. Clauset, M. E. Newman, and C. Moore. Finding community structure in very large networks. Physical review E, 70(6):066111, 2004.

[27] Q. Cui, M. Ward, E. Rundensteiner, and J. Yang. Measuring data abstraction quality in multiresolution visualizations. IEEE Transactions on Visualization and Computer Graphics, 12(5):709-716, 2006.

[28] W. Cui, X. Wang, S. Liu, N. H. Riche, T. M. Madhyastha, K. L. Ma, and B. Guo. Let it flow: a static method for exploring dynamic graphs. In 2014 IEEE Pacific Visualization Symposium, pp. 121-128. IEEE, 2014.

[29] A. Dal Col, P. Valdivia, F. Petronetto, F. Dias, C. T. Silva, and L. G. Nonato. Wavelet-based visual analysis of dynamic networks. IEEE transactions on visualization and computer graphics, 24(8):2456-2469, 2017.
[30] S. Diehl and C. Görg. Graphs, they are changing. In International Symposium on Graph Drawing, pp. 23-31. Springer, 2002.

[31] N. Elmqvist, T.-N. Do, H. Goodell, N. Henry, and J.-D. Fekete. Zame: Interactive large-scale graph visualization. In 2008 IEEE Pacific visualization symposium, pp. 215-222. IEEE, 2008.

[32] N. Elmqvist and J.-D. Fekete. Hierarchical aggregation for information visualization: Overview, techniques, and design guidelines. IEEE Transactions on Visualization and Computer Graphics, 16(3):439-454, 2010.

[33] P. Federico, J. Pfeffer, W. Aigner, S. Miksch, and L. Zenk. Visual analysis of dynamic networks using change centrality. In Proceedings of the 2012 International Conference on Advances in Social Networks Analysis and Mining (ASONAM 2012), pp. 179-183. IEEE Computer Society, 2012.

[34] T. M. Fruchterman and E. M. Reingold. Graph drawing by force-directed placement. Software: Practice and experience, 21(11):1129-1164, 1991.

[35] P. Goyal, S. R. Chhetri, N. Mehrabi, E. Ferrara, and A. Canedo. Dynamicgem: A library for dynamic graph embedding methods. arXiv preprint arXiv:1811.10734, 2018.

[36] P. Goyal and E. Ferrara. Graph embedding techniques, applications, and performance: A survey. Knowledge-Based Systems, 151:78-94, 2018.

[37] S. Hadlak, H.-J. Schulz, and H. Schumann. In situ exploration of large dynamic networks. IEEE Transactions on Visualization and Computer Graphics, 17(12):2334-2343, 2011.

[38] S. Hadlak, H. Schumann, C. H. Cap, and T. Wollenberg. Supporting the visual analysis of dynamic networks by clustering associated temporal attributes. IEEE Transactions on Visualization and Computer Graphics, 19(12):2267-2276, 2013.

[39] M. Harrower and C. A. Brewer. Colorbrewer. org: an online tool for selecting colour schemes for maps. The Cartographic Journal, 40(1):2737, 2003.

[40] C. Healey and J. Enns. Attention and visual memory in visualization and computer graphics. IEEE transactions on visualization and computer graphics, 18(7):1170-1188, 2012.

[41] N. Henry and J.-D. Fekete. Matrixexplorer: a dual-representation system to explore social networks. IEEE transactions on visualization and computer graphics, 12(5):677-684, 2006

[42] W. Javed and N. Elmqvist. Stack zooming for multifocus interaction in skewed-aspect visual spaces. IEEE transactions on visualization and computer graphics, 19(8):1362-1374, 2012.

[43] T. Kamada, S. Kawai, et al. An algorithm for drawing general undirected graphs. Information processing letters, 31(1):7-15, 1989.

[44] N. Kerracher, J. Kennedy, and K. Chalmers. A task taxonomy for temporal graph visualisation. IEEE transactions on visualization and computer graphics, 21(10):1160-1172, 2015.

[45] A. Kerren, A. Ebert, and J. Meyer. Human-Centered Visualization Environments: GI-Dagstuhl Research Seminar, Dagstuhl Castle, Germany, March 5-8, 2006, Revised Papers, vol. 4417. Springer, 2007.

[46] S. Kumar, W. L. Hamilton, J. Leskovec, and D. Jurafsky. Community interaction and conflict on the web. In Proceedings of the 2018 World Wide Web Conference on World Wide Web, pp. 933-943. International World Wide Web Conferences Steering Committee, 2018.

[47] Y. Liu, T. Safavi, A. Dighe, and D. Koutra. Graph summarization methods and applications: A survey. ACM Computing Surveys (CSUR), 51(3):1-34, 2018.

[48] Y. A. Malkov and D. A. Yashunin. Efficient and robust approximate nearest neighbor search using hierarchical navigable small world graphs. IEEE transactions on pattern analysis and machine intelligence, 2018.

[49] J. Moody, D. McFarland, and S. Bender-deMoll. Dynamic network visualization: Methods for meaning with longitudinal network movies. Retrieved November, 11:2008, 2004.

[50] A. Narayanan, M. Chandramohan, R. Venkatesan, L. Chen, Y. Liu, and S. Jaiswal. graph2vec: Learning distributed representations of graphs. arXiv preprint arXiv:1707.05005, 2017.

[51] M. Newman. Networks. Oxford university press, 2018.

[52] C. Nobre, M. Meyer, M. Streit, and A. Lex. The state of the art in visualizing multivariate networks. In Computer Graphics Forum, vol. 38, pp. 807-832. Wiley Online Library, 2019.

[53] A. Paranjape, A. R. Benson, and J. Leskovec. Motifs in temporal networks. In Proceedings of the Tenth ACM International Conference on Web Search and Data Mining, pp. 601-610, 2017.

[54] H. C. Purchase, E. Hoggan, and C. Görg. How important is the mental map?-an empirical investigation of a dynamic graph layout algorithm. In International Symposium on Graph Drawing, pp. 184-195. Springer, 2006. 
(C) 2020 IEEE. This is the author's version of the article that has been published in IEEE Transactions on Visualization and Computer Graphics. The final version of this record is available at: $x x . x x x x / T V C G .201 x . x x x x x x x /$

[55] B. Rozemberczki, O. Kiss, and R. Sarkar. Karate Club: An API Oriented Open-source Python Framework for Unsupervised Learning on Graphs. In Proceedings of the 29th ACM International Conference on Information and Knowledge Management (CIKM '20). ACM, 2020.

[56] S. Rufiange and M. J. McGuffin. Diffani: Visualizing dynamic graphs with a hybrid of difference maps and animation. IEEE Transactions on Visualization and Computer Graphics, 19(12):2556-2565, 2013.

[57] H.-J. Schulz and C. Hurter. Grooming the hairball-how to tidy up network visualizations? In INFOVIS 2013, IEEE Information Visualization Conference, 2013.

[58] B. Shneiderman. The eyes have it: A task by data type taxonomy for information visualizations. In The Craft of Information Visualization, pp. 364-371. Elsevier, 2003.

[59] C. Tominski, G. Fuchs, and H. Schumann. Task-driven color coding. In 2008 12th International Conference Information Visualisation, pp. 373380. IEEE, 2008.

[60] B. Tversky, J. B. Morrison, and M. Betrancourt. Animation: can it facilitate? International journal of human-computer studies, 57(4):247262, 2002.

[61] S. van den Elzen, D. Holten, J. Blaas, and J. J. van Wijk. Reducing snapshots to points: A visual analytics approach to dynamic network exploration. IEEE transactions on visualization and computer graphics, 22(1):1-10, 2016.

[62] S. Verma and Z.-L. Zhang. Hunt for the unique, stable, sparse and fast feature learning on graphs. In I. Guyon, U. V. Luxburg, S. Bengio, H. Wallach, R. Fergus, S. Vishwanathan, and R. Garnett, eds., Advances in Neural Information Processing Systems 30, pp. 88-98. Curran Associates, Inc., 2017.

[63] Y. Wang, D. Archambault, H. Haleem, T. Moeller, Y. Wu, and H. Qu. Nonuniform timeslicing of dynamic graphs based on visual complexity. In 2019 IEEE Visualization Conference (VIS), pp. 1-5. IEEE, 2019.

[64] P. C. Wong, P. Mackey, K. A. Cook, R. M. Rohrer, H. Foote, and M. A. Whiting. A multi-level middle-out cross-zooming approach for large graph analytics. In 2009 IEEE Symposium on Visual Analytics Science and Technology, pp. 147-154. IEEE, 2009.

[65] J. Xu, Y. Tao, Y. Yan, and H. Lin. Exploring evolution of dynamic networks via diachronic node embeddings. IEEE transactions on visualization and computer graphics, 2018.

[66] D. Zhang, J. Yin, X. Zhu, and C. Zhang. Network representation learning: A survey. IEEE transactions on Big Data, 2018.

[67] M. Zinsmaier, U. Brandes, O. Deussen, and H. Strobelt. Interactive levelof-detail rendering of large graphs. IEEE Transactions on Visualization and Computer Graphics, 18(12):2486-2495, 2012. 